\title{
Spectrum Sensing Issues and Techniques of Cognitive Radio Systems: A Review
}

\author{
Komal Arora, Shippu Sachdeva* and Manoj Sindhwani \\ Department of ECE, Lovely Professional University, Phagwara, Punjab - 144411, India; \\ manoj.16133@|pu.co.in
}

\begin{abstract}
In today's era of wireless and mobile communication, there is unaccustomed increase in number of subscribers which has led to demand of higher data rates. To support higher data rates, enormous bandwidth is required. As the spectrum is limited, problem of bandwidth scarcity has come into picture. Various techniques have been devised to resolve this problem. Cognitive Radio is one of them. Cognitive radio is the name given to the technology which senses the spectrum of primary users and allow the secondary users to send the data if primary users are not sending. Spectrum sensing is the major challenge of CR systems. This paper focuses on the challenges involved in CR systems and various spectrum sensing techniques.
\end{abstract}

Keywords: Carrier Sense Multiple Access (CSMA), Cognitive Radio (CR), Signal to Noise Ratio (SNR)

\section{Introduction}

Cognitive Radio (CR) is now a rising technology for the next generation of wireless communication system like 5th Generation (5G). The need of providing different services to different users has led to the demand of high spectrum bandwidth, but the spectrum is a limited resource. So the real problem is not of limited spectrum but of underutilized spectrum. The CR aims to resolve the problem of bandwidth scarcity by properly utilizing the unused bands of licensed users. FCC provides licenses to the users for the spectrum use. As these licensed users i.e. primary users don't use the spectrum bands all the time. So vacant bands can be sensed using spectrum sensing techniques in Cognitive Radio. CR performs four important tasks of spectrum sensing, managing the spectrum, spectrum sharing and spectrum mobility ${ }^{1,2}$. Spectrum sensing detect void spaces in the licensed bands. Spectrum management manages the time for which these void spaces can be used without causing any delay in transmission for primary user signal. Spectrum sharing helps to establish the uninterrupted conversation while shifting to better frequency band.

Characteristics of CR include Cognitive capability whereas Cognitive capability is the ability of CR to collect the information about the channel environment.

\section{Challenges Associated in Spectrum Sensing}

\subsection{Random Nature of Channel}

Wireless Channel is characterized by Multipath Fading; this may vary the strength of received signal which will increase the probability of wrong detection. Due to Fading observed by secondary users, there can be hidden Primary User Problem which is similar to the hidden node problem in Carrier Sense Multiple Accessing (CSMA). Hidden user problem specifies the problem in which the $\mathrm{CR}$ system creates undesired noise to the licensed receiver

${ }^{*}$ Author for correspondence 
as the licensed sender signal can't be checked due to the positions of CR devices. To avoid this, sensitivity of CR system should be sufficiently high and to achieve high sensitivity, Cooperative sensing in which multiple CR systems are incorporated is used. In this sensing, several $\mathrm{CR}$ systems share their individual channel measurements.

\subsection{Random Nature of Noise}

Minimum SNR is required by the system to detect the primary signal. So, variation in Noise estimation can adversely affect the system performance. This parameter is affected by thermal noise and calibration errors. Noise power is then chosen as the worst case noise assumption.

\subsection{Multiple Systems}

Several Cognitive Radios working in same frequency band of primary users severely affect the process of spectrum sensing. This increases the probability of wrong detection and the need of more accurate detector. So, interference limit is set very precisely ${ }^{1,9}$.

\subsection{Hardware Constraint}

The spectrum sensing method requires high precision Analog to Digital Converters (ADCs) with large dynamic range and high speed digital signal processors. For optimal detection at receiver, Noise variance estimation techniques are extensively used. The receivers must be capable of processing the narrowband baseband signals so as to get low complexity and low power processors. While Transmission, CR needs to sense the wider frequency band so that no opportunity is missed to detect white spaces. This need of analyzing a larger band leads to the additional requirement on the radio frequencies $(\mathrm{RF})$ components like antennas and power amplifiers.

\subsection{Sensing Spread Spectrum Primary Users}

Wireless devices operate either on fixed frequency or on spread spectrum. Fixed frequency devices include IEEE 802.11a/g based WLAN. Spread spectrum devices. The two types of Spread spectrum signals are FHSS and DSSS. FHSS based devices vary their working frequencies periodically to many narrowband channels. This variation process is called as hoping. The hoping pattern is decided on the basis of $\mathrm{p}-\mathrm{n}$ sequence which is known to both source and destination. Detecting Primary users using spread spectrum signaling is a very difficult task. The reason for this is that even if the actual bandwidth of primary user is much narrower but the power of primary user is spread over much wider bandwidth $\frac{12}{}$.

\subsection{Sensing Time period and Frequency}

Licensed users can vary their operating frequencies anytime while CR systems are operating on their own bands. To prevent interference to and from primary licensed band users, cognitive radio systems should be quick enough to detect the presence of licensed users as fast as possible. Also, they should leave the occupied band at the same time. So, there should be a fixed time period to sense the presence of primary users. If the characteristics of primary users are known to vary slowly, sensing frequency requirements can be somewhat relaxed. Sensing TV signals is a good example. Along with sensing frequency, other parameters the channel detection time, also affect the performance. Interference tolerance of primary license owners also affects the spectrum sensing frequency. Sensing time duration should also be optimum so as to keep an eye on the used channels and search for a vacant channel. Thus aim is to maximize system throughput while limiting interference. One procedure to achieve this is to utilize the guard bands of OFDM symbols and replace this time slots by spectrum sensing slots. So, it also saves useful Bandwidth. To decrease the sensing time, only varying patterns of spectrum must be sensed instead of sensing whole spectrum ${ }^{11}$.

\subsection{Decision Fusion in Cooperative Sensing}

Cooperative Sensing requires exchanging the data between cognitive radios and summing the outputs from different computation. The decisions made by each cognitive device can be hard or soft. The soft decision technique is better than hard decision procedure in settling the probability of missed detection. While hard decisions are found to complete as better as soft decisions when the number of cooperating users is high. Various diversity schemes can be used to fuse the results from multiple CR systems $\frac{3,4}{4}$.

\subsection{Security}

In cognitive radio, a FALSE user can modify its air interface to interpret as a primary user called as primary user emulation attack. The location of the transmitter is used for identifying an intruder. But the major problem is to develop effective solutions once an attack is identified ${ }^{2}$. 


\section{Cognitive Radio Spectrum Sensing Methods}

Various methods are proposed for identifying the existence of signal transmissions. So here we are explaining some spectrum sensing techniques, which are very common, and then comparison between the techniques will be discussed.

\subsection{Energy Detector based Sensing}

This is one of the simplest and traditional techniques used for spectrum sensing. This technique has the lowest complexity as it does not require pre- determined knowledge of licensed user's signal. In this sensing technique energy of the received signal is calculated and then compared with a threshold, it decides that primary user's signal is present. Performance metrics used are false alarm probability and missed detection probability. Problems encountered involve ${ }^{8}$. The method used for selecting threshold and their degrading performance in low SNR values. The performance of energy detection can be improved by using cubing operation instead of doubling operation. Also the use of double threshold in energy detector further improves the performance. In addition, adaptive threshold increases the detection probability in CR systems.

Cubing is the best option than squaring operation ${ }^{3,4}$. Further performance can be improved by using double threshold ${ }^{6,7}$. The block diagram of energy detector for reference is given below.

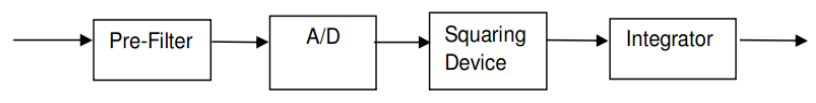

Figure 1. Block diagram of energy detector.

\subsection{Waveform-based Sensing}

Waveform based sensing is a technique in which predetermined patterns are used and synchronization is done in wireless systems, These patterns can be utilized for spectrum sensing by comparing the received signal with the saved copy. This sensing technique is considered to be better than energy detector based sensing. This technique is utilized only for the known signals which can be considered as the drawback. The advantage of the technique includes its reliability and also the performance improves with the increase in the length of predetermined sequences ${ }^{1}$.

\subsection{Cyclo Stationarity based Sensing}

OFDM signals are varied prior to transmission so as to produce particular frequencies in cyclic fashion at certain frequencies. This feature of OFDM is used for sensing of licensed user. This feature detection method is known as cyclo stationarity based sensing. Features used in detection can be power spectral density, mean correlation. The number of features can be added which will improve the performance of sensing algorithms in fading environments. But this will increase the supervisory information which will decrease the bandwidth efficiency.

\subsection{Radio Identification based Sensing}

The Vacant bands of primary users can be known by knowing the transmission strategies used by licensed users. This provides CR the knowledge about all the dimensions with higher precision. If primary user is utilizing Bluetooth technology of $2.4 \mathrm{GHz}, \mathrm{CR}$ uses this knowledge to interact with some known systems. The CR performs this task by either monitoring other modes while operating in a certain mode. In all these sensing, several features like cyclic prefix in OFDM are retrieved from the received signal and this feature can be extracted by comparing the last part of symbol with initials bits of symbol.

\subsection{Matched Filtering}

Matched Filter is a filter which aims to improve the performance by increasing SNR. The filter uses pre-determined knowledge of licensed user. It works by correlating the unknown signal with reference. Advantage of this technique is that the probability of false detection is achieved in short time. But the sample size is very large for a given false alarm probability for low SNR. Drawback is that primary users signalling features such as bandwidth, modulation used and frame format, $\mathrm{CR}$ requires receivers for different signal types, spectrum sensing implementation complexity is very high. Clear knowledge of every licensed signal is needed. If the knowledge is not precise, MF performance degrades. Also it requires a dedicated receiver for each licensed user ${ }^{5}$.

\subsection{Comparison of Various Sensing Methods}

Various spectrum sensing techniques are compared. It has been observed that the energy detection is the simplest algorithm for spectrum sensing but it can't be used for 
wide band signals. Matched filter works well in Gaussian noise and maximizes SNR, but it needs the perfect synchronization and initial information of licensed user signal. Cyclo stationarity detection performs well under high noise conditions, but its complexity is high. Hence, energy detection is utilized as it has lesser computational complexities ${ }^{1,13,14}$.

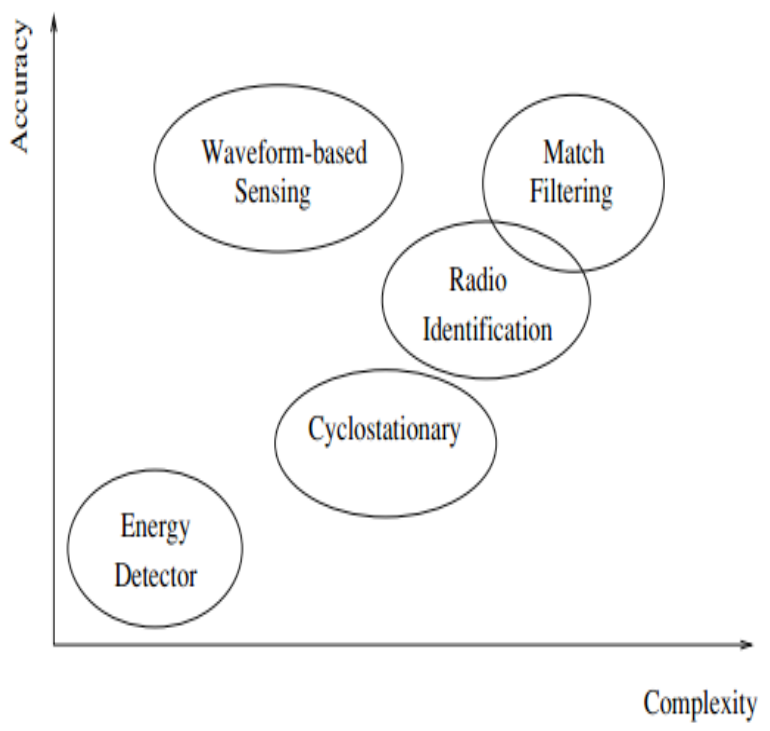

Figure 2. Comparison of spectrum sensing techniques.

\section{Conclusion}

Cognitive radio is a dynamic spectrum management technique used in wireless communication. This paper presents a vast comparison of spectrum sensing techniques. As spectrum scarcity is the main problem resolved by Spectrum sensing techniques. The most challenging task of CR system is Spectrum Sensing. The paper also focuses on the various issues involved and spectrum sensing techniques. We observed that energy detection is widely used because of low complexity. Future scope can be Adaptive threshold variation in co-operative sensing based MIMO CR system and in cubing operation based Energy detection.

\section{References}

1. Tevfik Yucek, Huseyin Arslan. a survey of spectrum sensing algorithms for cognitive radio applications. IEEE Communications Surveys \& Tutorials. Mar 2009; 11(1).
2. Khan A, Rehmani MH, Reisslein M. Cognitive Radio for Smart Grids: Survey of Architectures, Spectrum Sensing Mechanisms, and Networking Protocols. IEEE Communications and Surveys. 2016; 18(1).

3. Sonika, K Arora. Performance analysis of cubing based energy detector with adaptive threshold in cognitive radio over fading channel. IJAER. p. 20683-692.

4. Ghosh S, Bhowmick A, Nallagonda S, Roy SD, Kundu S. Performance of Weighted Fusion based Spectrum Sensing under Double Threshold in Cognitive Radio Network. 2016 International Conference on Microelectronics, Computing and Communications (MicroCom). Durgapur. 2016. p.1-4.

5. Igbinosa IE, Oyerinde OO, Srivastava VM, Mneney S. Spectrum sensing methodologies for cognitive radio systems: A Review. IJACSA. 2015; 6(12).

6. Arora K, Kansal A, Singh K. Comparison of energy detection based spectrum sensing methods over fading channels in cognitive radio. SPIJ. 2011; 5(12).

7. Xie S, Shen J, Liu L. Optimal threshold of energy detection for spectrum sensing in cognitive radio. Wireless Communications \& Signal Processing. 2009. WCSP 2009. International Conference on, Nanjing. 2009. p. 1-5.

8. Jain, Deepika, Kaur A, Ahuja S. Enhanced cognitive radio energy detection technique based on estimation of noise uncertainty. IJARCET. May 2016; 5(5).

9. Liu, Peng, et al. Full duplex joint sensing for opportunistic access in spectrum-heterogeneous cognitive radio networks. Apr 2011; 3(4).

10. Ghasemi A, Sousa E. Optimization of spectrum sensing for opportunistic spectrum access in cognitive radio networks. In Proceedings IEEE Consumer Communication and Networking Conference. Las Vegas, Nevada, USA. Jan 2007. p. 1022-26.

11. Khambekar N, Dong L, Chaudhary V. Utilizing OFDM guard interval for spectrum sensing. In Proc. IEEE Wireless Communication and Networking Conf, Hong Kong. Mar 2007. p. 38-42.

12. Datla D, Rajbanshi R, Wyglinski AM, Minden GJ. Parametric adaptive spectrum sensing framework for dynamic spectrum access networks. In Proc. IEEE International Symposium on New Frontiers in Dynamic Spectrum Access Networks, Dublin, Ireland; Apr 2007. p. 482-85.

13. Hu W, Willkomm D, Abusubaih M, Gross J, Vlantis G, Gerla M, Wolisz A. Dynamic frequency hopping communities for efficient IEEE 802.22 operation. IEEE Communication Mag. May 2007; 45(5):80-7.

14. Visotsky E, Kuffner S, Peterson R. On collaborative detection of TV transmissions in support of dynamic spectrum sharing. In Proceedings of IEEE International Symposium on New Frontiers in Dynamic Spectrum Access Networks, Baltimore, Maryland, USA. Nov 2005. p. 338-45. 